\title{
Distributional dynamics of life satisfaction in Europe
}

\author{
Vanesa Jordá* \\ jordav@unican.es \\ Telf. +34 942202275; Fax. +34942201603 \\ Borja López-Noval \\ borja.lopeznoval@unican.es \\ José María Sarabia \\ sarabiaj@unican.es \\ Santander, Spain.
}

Department of Economics, University of Cantabria. Av. los Castros SN, 39005

\section{Acknowledgements}

The authors acknowledge the financial support of the Ministerio de Economía y Competitividad (project ECO2016-76203-C2-1-P). We are also grateful to José Manuel Alonso, Jacques Silber, Martyna Kobus, and participants of the XXII Meeting of Public Economics and the Sixth ECINEQ meeting for their valuable comments. We also thank the editor and two anonymous referees for their helpful comments and suggestions.

\footnotetext{
* Corresponding author.
} 


\begin{abstract}
The promotion of subjective well-being is becoming a central goal of social and public policy. In this regard, it is sometimes argued that subjective well-being inequality is an informative indicator of social tensions. In this paper, we investigate the evolution of the life satisfaction distribution in Europe since 1973 using data from the Eurobarometer surveys. In order to respect the ordinal nature of subjective wellbeing and to avoid the need to impose an arbitrary scale, we use the Abul Naga and Yalcin index. We demonstrate that this index can be characterised as a measure of both inequality and polarisation, depending on the value of the parameters. We find that, at the European level, life satisfaction inequality was significantly higher in 2014 than in 1995 . This result is mainly explained by the increase in inequality in the Mediterranean countries and Ireland in recent years, but especially since the Great Recession. Although polarisation and inequality present a similar trend at the European level, some differing patterns are observed for particular countries, thus suggesting that these two phenomena are not only conceptually different, but also complementary in the analysis of the distribution of subjective well-being.
\end{abstract}




\section{Introduction}

As established in the Treaty on European Union (EU), the promotion of individual well-being is one of the fundamental aims of the EU. It is now well accepted that the notion of well-being goes beyond income (Stiglitz et al., 2009), although no consensus has been reached with regard to its measurement. Notwithstanding long-standing debates regarding the approaches and the indicators that might provide a valid alternative to the GDP per capita (see Decancq et al. (2015) for a review), self-assessed life satisfaction is considered one of the main pillars to evaluate individual well-being. In this sense, the Communication from the European Commission to the Council and the European Parliament (2009) considers, among other aspects, life satisfaction as one of its relevant dimensions, and more importantly though, it highlights the importance of attending to distributional aspects as indicators of social and economic cohesion.

Despite its relevance, there is scant evidence on the evolution of the distribution of subjective well-being. Most studies focused on the determinants of happiness inequality (Niimi, 2016; Ott, 2005; Ovaska and Takashima, 2010) or on the relationship between distributional patterns of income and subjective wellbeing (Alesina et al., 2004; Schneider, 2015), but just few analyses evaluated the level of happiness inequality. Veenhoven $(2005,2011)$ studied the evolution of the standard deviation of subjective wellbeing for the period 1973-2010 and observed that it has declined in all countries but West Germany and Portugal. This finding is consistent with new evidence found by Becchetti et al. (2014) who used the German Socio-Economic Panel database to study changes in the distribution of life satisfaction along the period 1991-2007. Stevenson and Wolfers (2008) focused on the distribution of self-reported happiness in the United States over the period 1972-2006. They observed a decreasing trend during the 70s and the 80 s, that reversed in subsequent years cancelling out about one third of previous decline.

A potential limitation of the aforementioned studies is that they used conventional inequality measures, thus considering subjective well-being as a cardinal variable. Despite there is some evidence that supports a cardinal interpretation of life satisfaction measures (Ferrer-i-Carbonell and Frijters, 2004), a conservative approach may consider subjective well-being as an ordinal variable (see e.g. Balestra and Ruiz, 2015; Madden, 2011). In addition, some surveys use verbal response scales, which makes especially disputable the use of conventional inequality measures. As discussed further in this paper, standard inequality measures are heavily dependent on the scale, and hence the evolution of disparities is sensitive to this choice.

Only few studies that focused on evaluating inequality in subjective well-being considered the ordinal nature of this variable. Madden (2011) conducted an analysis for Ireland that investigated the evolution of disparities in different measures of self-reported satisfaction over the period 1991-2004. It was the first study that assessed inequality in happiness using measures specifically designed for ordinal variables, finding a substantial fall in all the studied domains of life satisfaction. Dutta and Foster (2013) developed an inequality index which does not require assuming cardinality. They used the proposed index to evaluate the distributional patterns of happiness in the US for the period 1972-2010. As Stevenson and Wolfers (2008), they observed a U-shaped trend. Balestra and Ruiz (2015) used the inequality index developed by Abul-Naga and Yalcin (2008) for ordinal variables to evaluate the inequality in subjective well-being in the OECD countries using the 2010 round of Gallup World Poll. Comparing their results with those resulting from using conventional scale-dependent inequality measures, these authors concluded that not considering the ordinal nature of self-reported well-being might lead to fairly different conclusions.

Happiness inequality has been characterised as an indicator of social tensions, thus becoming crucial for policy purposes (Veenhoven, 2005; Becchetti et al., 2014). In this regard, Apergis and Georgellis (2015), focusing on the case of the $\mathrm{EU}$, assert that "happiness convergence could also be a legitimate policy goal and a useful barometer of changes in the political landscape, societal values, and citizens' sentiments about further European Integration" (Apergis and Georgellis, 2015, p. 68). Notwithstanding the role played by disparities as a source of social tensions, it is well known that polarisation is key in the emergence of social conflicts. Indeed, a society with low inequality levels, but at the same time split up in 
two well-defined classes with opposed interests (say rich and poor), might present serious conflicts, being the distance between these two groups the main source of social tension (Esteban and Ray, 1999). Although polarisation may be a more important cause of conflict than inequality, only Madden (2011) investigated polarisation patterns of life satisfaction.

The main contribution of this paper to the literature on the distribution of life satisfaction is twofold. First, we distinguish between inequality and polarisation in ordinal variables, both theoretically and empirically. We also demonstrate that the Abul Naga and Yalcin's (2008) measure, originally proposed as an inequality index, can be characterised as a measure of polarisation for a particular range of the parameters. This property makes this class of indicators especially appealing for the analysis of distributional dynamics of life satisfaction. Second, we study the evolution of the distribution of life satisfaction in Europe from 1973 until 2014, using data from the Eurobarometer surveys. To the best of our knowledge, this is the first analysis that explores the distributional patterns of life satisfaction in the Europe for such a long period of time. Furthermore, we cover the Great Recession and provide some insights on how the financial crisis has affected happiness inequality. In order to address inequality and polarisation trends, we use the theoretical framework developed in the front end of the paper. The index proposed by Abul Naga and Yalcin (2008) is scale independent, thus avoiding the need to impose an arbitrary scale. These results are then compared with the evolution of conventional inequality measures used by previous studies to evaluate the levels of life satisfaction inequality.

The rest of the paper is organised as follows. We begin by discussing the concepts and measurement of life satisfaction, before explaining the technical issues involved in the measurement of inequality and polarisation of ordinal variables. Thereafter, we present and discuss our main empirical findings. We conclude the paper by considering the implications of our study.

\section{Measuring inequality and polarisation in subjective well-being}

Life satisfaction of individuals is gauged by means of survey questions whose answers are categorised in a number of different states. Responses have an ordinal structure: the higher the category the higher the level of life satisfaction. Despite there is some evidence supporting a cardinal interpretation of life satisfaction measures (Ferrer-i-Carbonell and Frijters, 2004), in this paper we adopt a conservative strategy treating life satisfaction assessments as an ordinal variable. Hence, we assume that life satisfaction assessments are a positive monotonic transformation of an underlying phenomenon of interest: the so-called cognitive subjective well-being, which entails a cognitive process in which individuals might take into account, besides current feelings, other persons' conditions, own past experience and expectations of the future (Frey and Stutzer, 2002). In addition, we assume that life satisfaction assessments are interpersonally and ordinally comparable. Therefore, contrary to the cardinal approach, we do not make any assumption as regards the relative difference between satisfaction answers, but only that all individuals do share a common interpretation of each possible answer (Ferrer-i-Carbonell and Frijters, 2004).

The analysis of inequality in subjective well-being from an ordinal approach requires using different measures from those conventionally employed for cardinal variables. Most conventional inequality measures take the mean as a reference point to measure the dispersion of the distribution. The use of these measures on ordinal variables requires defining a scale, thus making the ranking of countries heavily dependent on that choice (Allison and Foster, 2004; Madden, 2010). In this regard, let us consider the following example in which we aim to compare two countries in terms of their average level of life satisfaction. The frequency distributions (the proportion of individuals in each category) of the life satisfaction variable with five possible categories, $f_{1}$ and $f_{2}$, are presented in Table 1. Applying the Cantril scale $(c)$, we would conclude that Country 1 is, on average, better off because its mean is higher than in Country 2. If we used instead the alternative scale $c$ ' this conclusion would be reversed, being the mean in Country 2 higher than in Country 1 . Consequently, the robustness and validity of inequality assessments using inequality measures that rely on the mean might be also affected by rescaling. Consider, for 
instance, the coefficient of variation of both distributions using those two scales. We observe that Country 1 would be more equal than Country 2 using the $c$ scale, whereas using the alternative scale ( $c$ '), Country 1 would present higher levels of inequality.

\section{[Table 1 HERE]}

The limitations of conventional measures to assess inequality in categorical variables make room for alternative approaches based on order statistics. ${ }^{1}$ In particular, the use of the median as a reference point seems to provide an appealing framework. The measure proposed by Blair and Lacy (2000) was one of the first attempts to obtain a median-based inequality index for ordinal variables. More recently, Allison and Foster (2004) developed a partial inequality ordering, which ranks distributions according to their spread to the median. Most of the proposals to measure disparities in ordinal variables are based on this ordering (Apouey, 2007; Abul-Naga and Yalcin, 2008; Kobus, 2014; Lazar and Silber, 2013), which makes them invariant to the numerical scale. ${ }^{2}$

There is some controversy about what phenomenon median-based statistics do actually measure. According to Zheng (2008), median-based inequality indices are indeed polarisation measures. For cardinal variables, bipolarisation is seen as a measure of the size of the middle class, which focuses on the concentration of the population at the two tails (see Foster and Wolfson, 2010). ${ }^{3}$ As we describe below, inequality indices for ordinal variables measure how concentrated the data is around the categories above and below the median. The most unequal distribution has, indeed, all the probability mass equally concentrated at the top and the bottom categories. It should be, however, noted that for categorical variables, the most unequal distribution can coincide with the most polarised one. This case is clearly observed for variables with only two categories. The variance is given by the product of the sample size, the probability of the first category (the bottom one) and the probability of the second category (the top one). Therefore, for a given sample size, the dispersion would be maximised when the probability is equally distributed in both categories, i.e. when there are two poles of equal size.

The previous example points out that the concepts of polarisation and inequality in ordinal variables are closely related. These two distributional aspects have been extensively studied in the case of cardinal variables, concluding that, despite being theoretically different, these two features of the distribution are also finely intertwined. To make a clear distinction between dispersion and polarisation in ordinal variables, we use the two basic properties that bipolarisation measures must satisfy (Foster and Wolfson, 2010; Wang and Tsui, 2000), namely increased spread and increased bipolarity. Following the literature on polarisation, we identify them with their corresponding orderings for categorical variables (See Appendix A for a technical description of these properties).

To illustrate these two properties, Figure 1 shows the distribution of a life satisfaction variable with five categories. In the left panel we assume that individuals are clustered in two categories: "not very satisfied" and "fairly satisfied", above and below the median category ("neither satisfied nor dissatisfied") respectively. Now let us assume that there is a relocation of the groups. All individuals that were "not

\footnotetext{
${ }^{1}$ Within this class, we find the interquartile range, which is scale dependent, but since it is based on the ranking of individuals, it is order preserving. The main problem of this measure is that it ignores a large amount of information contained between the two quartiles.

${ }^{2}$ See Cowell and Flachaire (2012) and Silber and Yalonetzky (2011) for alternative inequality measures for ordinal variables.

3 The concept of bipolarisation is associated with the concentration of the population in two poles, whereas polarisation considers a clustering of the population around an arbitrary number of poles. Socio-economic polarisation (Zhang and Kanbur, 2001) evaluates the differences between socially predefined groups by ethnicity, religion, etc., and the within-country variation of these groups. A decrease of the distance between the different subgroups would reduce socio-economic polarisation because it narrows the gap between the different poles. On the contrary, as the within-group variation declines, the groups become more homogeneous and, hence, socio-economic polarisation increases. Although these concepts are closely related, the underlying phenomena that they measure may present contrasting evolutions (see Duclos and Taptué, 2014).
} 
very satisfied" become "not at all satisfied" and the group of population that was "fairly satisfied", becomes "very satisfied". These movements increase the spread of the distribution from the median, and consequently both bipolarisation and inequality would increase. Figure 1 (right panel) illustrates graphically an increase of bipolarity in the case of categorical variables. Below the median all individuals are "not very satisfied" with their life, and above the median, there is an equal proportion of individuals that are "satisfied" and "very satisfied" and no one is "fairly satisfied". If these two groups above the median cluster at the category "fairly satisfied", inequality decreases because the dispersion above the median is lower, while bipolarisation goes up because the movement of probability mass has resulted in the emergence of a new pole.

\section{[Figure 1 HERE]}

For ordinal variables, Allison and foster (2004) developed a partial ordering (henceforth, the AFordering) to rank distributions according to their spread from the median, whereas the increased bipolarity (IB) property is characterised by the IB-ordering developed by Apouey (2007). The inequality measures developed in the literature (Blair and Lacy, 2000; Allison and Foster, 2004; Abul Naga and Yalcin, 2008) satisfy the partial AF-ordering, but polarisation measures should verify also the IB-ordering. Indeed, the IB property is what distinguishes bipolarisation from inequality, since a progressive transfer between individuals that are both above or below the median would reduce inequality while increasing polarisation (Foster and Wolfson, 2010).

Abul Naga and Yalcin (2008) relied solely on the partial AF-ordering to construct the following family of inequality indices (henceforth the ANY index) which is invariant to scale:

$$
I_{\alpha, \beta}=\frac{\sum_{j<m}\left(F_{j}\right)^{\alpha}-\sum_{j \geq m}\left(F_{j}\right)^{\beta}+J+1-m}{\kappa_{\alpha, \beta}+J+1-m},
$$

where $\kappa_{\alpha, \beta}=(m-1)(1 / 2)^{\alpha}-\left(1+(J-m)(1 / 2)^{\beta}\right)$ and $J$ is the number of categories. $\alpha$ and $\beta$ are two parameters which enable the analyst to vary the weight given to differences in life satisfaction below and above the median. Because the choice on the value of these parameters belongs to the researcher, it is essential to understand the potential implications of this decision. $I_{\alpha, \beta}$ satisfies the AF-ordering for any positive value of parameters $\alpha$ and $\beta$ (see Lemma 4 in Abul Naga and Yalcin (2008)). However, the range of the parameters was restricted for simplicity, so that the measure was originally defined for parameter values higher or equal to 1. According to Abul Naga and Yalcin (2008), the combination of convex (parameter values higher than 1) and concave (parameter values between 0 and 1) functions would make it difficult to determine the sensitivity of the index to specific parts of the distribution. However, this choice on the parameters has specific implications regarding the consideration of IB movements. For parameter values strictly higher than 1, the ANY measure increases due to IB movements below the median, but decreases if this kind of transfers takes place above the median. For the limiting case given by the so-called absolute index $(\beta=\alpha=1)^{4}$, IB movements leave the measure unchanged. The ANY measure is, therefore, characterised as an index of polarisation when $\alpha>1$ and $\beta \in(0,1)$ (see Appendix A, Proposition 1).

Let us illustrate the behaviour of this measure with an example. Table 2 presents three hypothetical distributions with seven categories which share a common median (the fourth category). $F_{2}$ and $F_{3}$ are obtained from $F_{1}$ by an IB movement below and above the median respectively. $A(0.5)$ refers to the Apouey (2007) measure which is an index of polarisation for ordinal variables which satisfies both the AF- and the IB-ordering. Respect to the baseline distribution $\left(F_{1}\right)$, this measure reports an increase in polarisation of the same amount for IB movements above and below the median because it is

\footnotetext{
${ }^{4}$ The absolute index would be the only case that holds the property of symmetry, i.e. the same importance is given to the distribution above and below the median. However, as this index is insensitive to IB movements, hence it might not be used to evaluate either inequality or polarisation.
} 
characterised by the property of symmetry. $I_{2,2}$ denotes the ANY measure when both parameters are set equal to 2, thus satisfying the restriction on the parameters originally proposed by Abul Naga and Yalcin (2008). We observe that the clustering below the median increases the value of this measure whereas the IB movement above the median decreases inequality.

\section{[Table 2 HERE]}

Although it may be debatable whether inequality measures for categorical variables should be consistent with the IB-ordering or not, the choice must be congruent for both parts of the distribution. In this regard, the restriction given in Proposition 1 (Appendix A) characterises the ANY measure as a polarisation index, so that $I_{2,0.5}$ increases due to IB movements above and below the median. By contrast, $I_{0.5,2}$ characterises the ANY measure as an inequality index. Hence, an IB-transfer makes the distribution more homogeneous in either side of the median, reducing inequality in that part of the distribution. As a result, as shown in the last column of Table 2, overall inequality would also fall.

The previous example highlights the flexibility of the ANY measure, not only because it allows us to ascribe different weights to differences below and above the median, but also because we can measure distinct distributional phenomena depending on the value of the parameters. The ANY index can be characterised as an inequality measure when $\alpha \in(0,1)$ and $\beta>1$. The ANY inequality measure becomes more sensitive to the top of the distribution as $\alpha$ tends to 0 for a given value of $\beta$, whereas it abstracts more from dispersion above the median as $\beta$ tends to infinity, for given values of the $\alpha$ parameter. On the other hand, the ANY polarisation measure $(\alpha>1$ and $\beta \in(0,1))$ becomes more sensitive to the top of the distribution as $\alpha$ tends to $\infty$ for a given value of $\beta$, whereas for a given $\alpha$, less weight is given to polarisation in the upper tail as $\beta$ tends to 0 .

\section{Distributional dynamics of life satisfaction in Europe}

\subsection{Data}

Life satisfaction is measured in the Eurobarometer surveys since 1973 by means of the question: "on the whole, are you very satisfied, fairly satisfied, not very satisfied or not at all satisfied with the life you lead?" The wording has remained unchanged over the period under analysis (1973-2014), which is essential to make the measure perfectly comparable on this respect. ${ }^{5}$ Diener (2006) distinguishes two possible meanings regarding life in the life satisfaction question: it may refer either to current life-as-awhole or to life since birth. The wording of the question in the Eurobarometer surveys ("[...] the life you lead [...]") points to the former meaning, although not explicitly. The measurement scale is not numerical, thus further supporting an ordinal approach. A nice feature of non-numerical scales is that they avoid individuals focusing at the extremes or the midpoint of the scale (Diener et al., 2013).

In order to have a more homogeneous group of countries and to increase the relevance of our results for policy purposes, we limit our sample to those countries sharing both a common political system and a monetary policy. Seven countries have participated in all Eurobarometer surveys, including, Belgium, the former West Germany, France, Ireland, Italy, Luxembourg, and The Netherlands. Greece joined the survey in 1980, Spain and Portugal in 1985, the former East Germany in 1990 and, finally, Austria and Finland in 1995.

A technical issue to be considered is the order of the questions in the survey, which is acknowledged to affect life satisfaction assessments. Most of the Eurobarometer surveys formulate the life satisfaction question at the beginning of the interview to avoid this problem. The answers of subjective surveys may

\footnotetext{
${ }^{5}$ Actually, since 2012 the survey distinguishes among individuals who do not answer the question and those that answer "I do not know", but given that only very few people choose this response category it does not seem a problem to simply omit them.
} 
also be shaped by the social nature of these surveys. Regarding life satisfaction self-assessments, it is observed that individuals especially predisposed to conform to social norms are less likely to report low levels of life satisfaction (see Diener et al., 2013; Easterlin, 1974). The fact that Eurobarometer interviews are conducted face-to-face makes these data more prone to this source of bias, a caveat that we should keep in mind when interpreting results.

Eurobarometer surveys usually targets 1,000 persons per country. ${ }^{6}$ As usual in surveys collecting data on life satisfaction, the response rate is remarkably high: always over 98 per cent. Country level measures are computed using national weights provided in the Eurobarometer surveys databases. For measures at the European level, national weights are transformed according to the population size of the country.

\subsection{Results}

In this section we analyse the evolution of the distribution of life satisfaction in Europe from 1973 to 2014. We first focus on regional trends in inequality and polarisation and then, we move onto the analysis at the country level to provide a detailed picture of the national patterns behind the observed evolution of the European distribution of subjective well-being. Figure 2 presents the evolution of inequality in life satisfaction in Europe from 1995 to 2014. We have calculated the ANY index for different combinations of the following parameter values $\alpha=0,0.25,0.5,0.75 ; \beta=1.5,4, \infty .{ }^{7}$ These values satisfy the constrain $\alpha \in(0,1)$ and $\beta>1$, hence characterising the ANY index as an inequality measure. The $95 \%$ confidence intervals have been calculated using the variance of the ANY measure obtained by means of the multivariate Delta method (see Appendix A, Statistical inference).

Our estimates reveal an increase in life satisfaction inequality since 1995 according to virtually all measures. From 1995 to 2008 the inequality levels remained, on average, stable, presenting only small variations that were counterbalanced in subsequent years. The observed increase is, therefore, the result of the upward trend observed since 2008, coinciding with the coming up of the Great Recession. The role of the economic dimension as a potential determinant of life satisfaction inequality has been already investigated by Madden (2011), who found that the distribution of different domains of life satisfaction became more equal during a period of economic boom in Ireland. This result has been confirmed by Becchetti et al. (2014), whose estimates suggested a negative relationship between per capita income and happiness inequality. As described below, the observed increase in inequality is principally explained by the rise in inequality levels in Spain, Greece, Italy and Ireland, countries that have been severely hit by the economic crisis. However, in most of those countries, inequality already presented an upward trend before the recession started. Hence, although it would be tempting to look for a single explanation of the observed patterns, the rise in life satisfaction inequality seems to be associated with multiple factors.

\section{[Figure 2 HERE]}

Regarding the sensitivity of the estimates of life satisfaction inequality to the choice on the weight given to inequality below and above the median, our results suggest that the trends are rather robust to the values of $\alpha$ and $\beta$. Only when $\alpha$ is set equal to zero, thus making the measure completely insensitive to the distribution below the median, the estimated inequality trend seems to differ from the distributional patterns reported by rest of the measures. By comparing both measures with $\alpha$ equal 0 with those that only consider disparities among the bottom two categories $(\beta=\infty),{ }^{8}$ we observe that disparities within the

\footnotetext{
${ }^{6}$ With the exception of Luxembourg that usually collects data of 300 individuals.

${ }^{7}$ The values of the $\alpha$ parameter have been chosen to cover the whole range of this parameter $(0,1)$. The $\beta$ parameter has been set to infinity, to present the evolution of the limiting measure; to 4 following Abul Naga and Yalcin (2008); and to 1.5 to analyse the evolution of inequality when more weight is given to disparities above the median. The results for the limiting case when $\alpha$ tends to 0 and $\beta$ tends to infinity have not been presented because it would correspond to a measure which neglects both inequality above and below the median.

${ }^{8}$ The median category in Europe is the third one during the period $1995-2014$. The life satisfaction variable used in this study involves four categories, hence the distribution below the median includes the first two, not at all satisfied and not very satisfied, and above the median it also comprises two categories, fairly satisfied and very satisfied.
} 
two parts of the distribution show virtually opposite trends during the whole period. The intermediate inequality measures $(\alpha=0.25,0.5$ and $0.75 ; \beta=1.5$ and 4$)$ present analogous trends to those observed for the bottom part. Because the ANY inequality measure is additively decomposable in disparities above and below the median (see Appendix, Eq. (A.3)), the evolution of overall inequality in life satisfaction might be mainly governed by disparities at the bottom of the distribution, except in the year 2004 when the increase of inequality above the median is not offset by the fall of inequality at the bottom of the distribution.

Turning now our attention to the evolution of polarisation, Figure 3 presents the evolution of the ANY measure in Europe for several combinations of the parameters that satisfy the condition $\alpha>1$ and $\beta \in(0$, 1). Polarisation trends mirror the evolution of inequality; life satisfaction seems to be more polarised in 2014 than in 1995 as a result of the raise in polarisation levels since 2008, an upward trend that is particularly evident for the distribution below the median. In line with the results regarding the evolution of inequality, polarisation below and above the median present opposite trends during the period analysed. Setting $\alpha$ equal to 1.5 , the evolution of polarisation exhibits fundamentally the same evolution as the distribution below the median. On the contrary, for $\alpha$ equal to 4 , the ANY polarisation measures present as similar pattern to the limiting case when $\alpha$ tends to infinity. When $\alpha$ is set equal to 4 the distribution below the median is barely considered because the frequency of the bottom categories is so small (less than 0.22 for all years) that, to the power 4 , becomes virtually irrelevant and hence the measure essentially captures polarisation among the top two categories. Thus, when $\beta$ tends to zero and $\alpha$ $=4$ the measure reports values close to zero and remains relatively stable because the distribution above the median is not considered and differences in life satisfaction below the median are of marginal importance.

\section{[Figure 3 HERE]}

An interesting fact is that these series seem to be much more volatile than the ones observed for inequality. As explained above, for the limiting case when $\alpha$ tends to infinity and for $\alpha$ equal to 4, the polarisation is essentially evaluated at the top two categories and hence, any spread away from the median implies a transfer of probability mass from the third to the fourth category. The third category presents a higher frequency than the fourth category, thus an increase in the spread would necessarily lead to an IB movement because it reduces the differences in the size of these two poles. As explained in Section 2, the fundamental difference between inequality and polarisation is that an IB movement would increase polarisation while reducing inequality. Thus, the effect of an increase (decrease) in the spread above the median on inequality is attenuated by the increase (decrease) in bipolarity, whereas its impact on polarisation is exacerbated by the IB movement. An analogous explanation justifies the higher volatility of polarisation measures that only consider the distribution below the median. On the contrary, those measures that evaluate polarisation at both sides of median $(\beta=1.5, \alpha=0.25,0.50,0.75)$ exhibit variations of similar size to the ones observed for inequality because, for four categories, an increased spread movement does not necessarily lead to an increase in bipolarity.

Thus far we have analysed the distribution of life satisfaction in Europe, which results from the aggregation of the different distributional patterns of the countries that conform this region. Now we focus on the evolution of inequality and polarisation trends at the country level. Figure 4 presents the evolution of the ANY index as a measure of both inequality and polarisation from 1995 to 2014 for the different countries. ${ }^{9}$ As regards inequality, we have calculated the ANY index for $\alpha=0.5, \beta=1.5$ and its two limiting cases: when only the bottom part is considered $(\alpha=0.5, \beta \rightarrow \infty)$ and when only changes above the median affect inequality $(\alpha \rightarrow 0, \beta=1.5)$. In order to compare these results with the time trend

\footnotetext{
${ }^{9}$ In Greece, the modal category changes from "fairly satisfied" to "not very satisfied" from 2011 onwards. Therefore, we only present estimates on inequality and polarisation until 2011 because the ANY measure is not comparable across distributions with different modal categories.
} 
of polarisation in life satisfaction, we have also computed the ANY measure for $\alpha=1.5, \beta=0.5$ and two indices that consider only the distribution below the median $(\alpha=1.5, \beta \rightarrow 0)$ and above the median $(\alpha \rightarrow$ $\infty, \beta=0.5) .{ }^{10} 95 \%$ confidence intervals have been calculated using the statistical inference developed in the Appendix.

Before moving onto the analysis of national trends in inequality and polarisation, it should be worth nothing that our interest resides in the ANY measures that consider the whole distribution: $\alpha=0.5, \beta=$ 1.5 for inequality (green line) and $\alpha=1.5, \beta=0.5$ for polarisation (light blue line). The cases that only consider inequality and polarisation above/below the median are presented to provide some insights about which part of the distribution is driving the observed trends when the whole distribution is considered. According to the evolution of life satisfaction inequality, we can identify a group of countries with low and quite stable levels of inequality along the analysed period: Austria, Belgium, West Germany, Finland and France. The observed stability in life satisfaction inequality is the result of offsetting movements observed along the whole series at both sides of the median of the distribution. Although polarisation seems to be much more volatile than inequality, both distributional phenomena present mirror evolutions. Indeed, as observed for Europe, only the measures that consider exclusively the distribution below or above the median present higher variability. As explained above, an increase in the spread of the distribution implies an IB movement when only two categories are considered on either side of the median if the category that is farther away from the median has a smaller frequency.

On the other hand, in the Netherlands, East Germany and Luxembourg inequality presents offsetting variations, which led to no significant change in the level of inequality. In the case of the Netherlands three differentiated phases are observed: downward trend from 1995 to 1999, that became positive in 2000 and then, after a modest decrease, stabilises in 2005 until the end of the period. East Germany and Luxembourg present unstable inequality levels that seem to fluctuate around the value 0.4 during the period 1995-2014. In East Germany and the Netherlands, the inequality trends for the bottom categories describe parallel patterns to those observed for the whole distribution, thus suggesting that the distribution below the median is the main cause of the observed evolution in life satisfaction inequality. This parallelism is not that evident in Luxembourg, where the top of the distribution governs the evolution of inequality from 1999 to 2001 and during the period 2012-2014. Polarisation and inequality present analogous evolutions in East Germany. In the Netherlands, although polarisation shows fairly similar patterns to those observed for inequality, during the period 2001-2005, polarisation remains roughly constant whereas inequality exhibits an ascending trend that reaches its peak in 2004. Luxembourg also presents different patterns between inequality and polarisation in certain years of the period 1998 to 2008 .

Finally, we have countries characterised by high and changing inequality levels: Spain, Greece, Ireland, Italy and Portugal. Among them, Spain and Greece show a common pattern: first, a downward trend is observed since 1995 until 2007, and then a sharp rise in inequality that virtually cancelled out the previous reduction and, in the case of Spain, led to higher inequality levels. The upward trend coincides with the beginning of the Great Recession, suggesting that the fall in disposable income and the escalation of unemployment rates might be two potential candidates for explaining the rise in inequality (Becchetti et al., 2014). The analysis of the changes at both sides of the median shows that the evolution of the whole distribution is basically determined by the trend observed in its lower part, which suffered the most the consequences of the last economic crisis that hit these countries particularly strongly. Although we acknowledge that it might just partially explain the ascending trend of life satisfaction inequality, this evidence suggest that the Great Recession has had an impact in the life satisfaction distribution in these countries.

\section{[Figure 4 HERE]}

\footnotetext{
${ }^{10}$ For the sake of clarity we only present few combinations of the parameter values. In Appendix B, we present the evolution of inequality and polarisation for other parameter values, which reveal parallel patterns to those presented in this section.
} 
Regarding Portugal, after a modest decline in inequality at the end of the 90s, inequality rose from 2000 until 2006, and then stabilised until the end of the period. Ireland and Italy show rather constant inequality levels before 2000, when inequality started to rise. The upward trend is rather smooth in the case of Ireland, whereas in Italy, inequality presents fluctuations around the observed ascending trend. The comparison of these trends with the inequality patterns at both sides of the median suggests that the evolution of the whole distribution in these three countries is basically determined by the trend observed in its lower part. Although polarisation presents more pronounced variations at the top of the distribution than inequality, the evolution of both phenomena is also fairly similar in Portugal and Italy. For Ireland, however, there are subtle differences between polarisation and inequality. The upward trend of inequality since 2005 is not observed in the case of polarisation, which shows quite stable levels until 2011. These differing trends suggest that the increase in the spread that boosted inequality levels also led to a decrease in bipolarity, thus keeping polarisation stable.

Our findings do not align with previous empirical evidence, which suggest that life satisfaction inequality fell in most of the European countries from 1973-2010 (Veenhoven, 2011). Prior research evaluated inequality levels by means of conventional inequality measures that require defining a scale for the life satisfaction variable, whereas our estimates respect its ordinal nature. The contrasting results might not only be explained by the use of a different indicator to measure disparities in life satisfaction, but also by the consideration of different time frames. Hence, to better appraise the potential implications of using conventional inequality measures to assess inequality in ordinal variables, we compare the evolution of the ANY inequality measure with two scale-dependent indices over the period 1973-2014.

Table 3 presents the absolute change (along with 95\% confidence intervals) and the growth rate of the ANY measure for different parameter values, the Gini index and the standard deviation. The standard deviation is one of the most popular measures used to evaluate inequality in life satisfaction (see e.g. Veenhoven (2011), Stevenson and Wolfers (2008)) along with the Gini index (Niimi, 2016), which, as the ANY measure, is bounded between 0 and 1. To transform the ordinal variable used to measure life satisfaction into a cardinal variable we use a Cantril scale. ${ }^{11}$ Before moving onto the analysis of these results, it is important to emphasise that our interest does not reside in comparing the absolute change in inequality. The estimated measures give different importance to the distribution above and below the median, which, may affect substantially the estimated level of inequality and, hence, its absolute change. Instead, we are concerned with the robustness of the long-term trend. Thus, we focus on the sign of the change and its statistical significance.

\section{[Table 3 HERE]}

The rates of change of the two limiting cases of the ANY measure that only consider the distribution below $(\alpha=0.5, \beta=\infty)$ and above $(\alpha=0, \beta=1.5)$ the median differ not only in the value, but also in the sign. As described previously, in most countries the bottom and the top categories present fairly different, and even opposite, evolutions. Greece is the only country where both parts of the distribution exhibit equivalent evolutions, which suggest a decline of inequality over the period under analysis. Despite the contrasting patterns depicted by the bottom and the top categories, the trends of the ANY indices that consider the whole distribution seem to be fairly robust to the value of the $\alpha$ and $\beta$ parameters. Our estimates suggest that inequality increased in West Germany, East Germany and the Netherlands and fell significantly in Austria, Belgium, Spain, Greece and Ireland. In these countries the change in inequality was not only statistically significant, but also economically relevant with increments ranged between 6 and 23 per cent and declines between 5 and 22 per cent. For the rest of the countries, the change in

\footnotetext{
${ }^{11}$ Using the Cantril scale is the simplest, although widely used, approach to transform ordinal variables into cardinal ones. See Van Doorslaer and Jones (2003) for a review on the different techniques adopted in the literature to define a scale for categorical variables.
} 
inequality does not seem to be significant. These results differ substantially from the evolution described by the scale-dependent measures, which only show significant declines of inequality in Spain, Ireland and Greece and, in the case of the standard deviation, a significant increase in East Germany. The change in inequality might not be different form zero for the other European countries.

\section{Conclusions}

With the upsurge of economic research on happiness, there have been an increasing number of studies focusing on the distribution of subjective well-being. Previous empirical evidence is mainly based on conventional inequality indices. The use of these measures implicitly conceives subjective well-being as a cardinal variable for which it is necessary to impose a particular scale. Standard inequality indices are scale dependent, so the levels and the trends in inequality might differ depending on the scale. Hence, the results of these analyses could be potentially misleading.

We have analysed the evolution of life satisfaction inequality and polarisation in Europe from 1973 to 2014. To avoid imposing an arbitrary scale to evaluate the levels of life satisfaction inequality, we have used the measure proposed by Abul Naga and Yalcin (2008), which is scale independent. We have demonstrated that this measure is able to assess both, polarisation and inequality, for specific ranges of the parameters. To do so, following Apouey (2007), we have characterised these two distributional phenomena for ordinal variables, concluding that, albeit closely related, these two features of the distribution are also conceptually different. Indeed, our results also point out that, though showing similar trends, polarisation and inequality present differing patterns in the Netherlands, Luxembourg and Ireland. Hence, our analysis illustrates that polarisation and inequality are not only theoretically different, but complementary in the analysis of distributional patterns of life satisfaction.

The main finding of this analysis is that life satisfaction inequality rose significantly in Europe over the period 1995-2014. The upward trend started in 2008, mainly underpinned by the increase in inequality in the Mediterranean countries and Ireland, thus suggesting that the deterioration of the labour market conditions and the contraction of disposable income during the Great Recession had a strong effect on the distribution of life satisfaction. Even though the existing evidence points towards a prominent role of the economic and labour domains in life satisfaction inequality (Becchetti et al., 2013), in most of those countries inequality already presented an upward trend before the recession started. Thus, although it would be tempting to look for a single explanation of the observed patterns, the rise in life satisfaction inequality seems to be associated with multiple factors, whose analysis might offer opportunities for further research.

To illustrate the potential implications of using scale-dependent measures to assess inequality in ordinal variables, we have compared the growth rate of the standard deviation and the Gini index with the relative change in the ANY measure, an index specifically designed to evaluate disparities in ordinal variables. Conventional inequality measures show significant changes in inequality for few countries, whereas the ANY index exhibits significant variations in most of the European countries analysed. These different results suggest that, as pointed out by previous studies, the choice between the ordinal and the cardinal approach is not neutral, and hence the use of conventional inequality measures might be problematic. 


\section{References}

Abul Naga, R. H., \& Yalcin, T. (2008). Inequality measurement for ordered response health data. Journal of Health Economics, 27(6), 1614-1625.

Alesina, A., Di Tella, R., \& MacCulloch, R. (2004). Inequality and happiness: are Europeans and Americans different?. Journal of Public Economics, 88(9), 2009-2042.

Allison, R. A., \& Foster, J. E. (2004). Measuring health inequality using qualitative data. Journal of Health Economics, 23(3), 505-524.

Apergis, N., \& Georgellis, Y. (2015). Does Happiness Converge?. Journal of Happiness Studies, 16(1), 67-76.

Apouey, B. (2007). Measuring health polarization with self- assessed health data. Health Economics, 16(9), 875-894.

Balestra, C., \& Ruiz, N. (2015) Scale-Invariant Measurement of Inequality and Welfare in Ordinal Achievements: An Application to Subjective Wellbeing and Education in OECD Countries. Social Indicators Research, 123(2), 479-500.

Becchetti, L., Massari, R., \& Naticchioni, P. (2014). The Drivers of Happiness Inequality: Suggestions for Promoting Social Cohesion. Oxford Economic Papers, 66(2), 419-442.

Blair, J., \& Lacy, M. G. (2000). "Statistics of ordinal variation". Sociological Methods \& Research, 28(3), 251-280.

Commission Of The European Communities (2009). GDP and Beyond: Measuring Progress in a changing world. Communication from the Commission to the Council and the European Parliament, 433. Brussels.

Cowell, F. A., \& Flachaire, E. (2012). "Inequality with ordinal data". In European Economic Association and Econometric Society Conference, Málaga.

Decancq, K., Fleurbaey, M. \& Schokkaert, E. (2015). "Happiness, Equivalent Incomes and Respect for Individual Preferences", Economica, 1-25.

Diener, ED (2006). Guidelines for National Indicators of Subjective Wellbeing and Ill-Being. Applied Research in Quality of Life, 1(2), 151-57.

Diener, Ed, Inglehart, R. \& Tay, L. (2013). Theory and Validity of Life Satisfaction Scales. Social Indicators Research 112(3), 497-527.

Duclos, J.Y., \& Taptué, A.M (2014) "Polarization". Available at: http://www.andremarietaptue.com/doc/polarization.pdf.

Dutta, I., \& Foster, J. (2013). Inequality of Happiness in the US: 1972-2010. Review of Income and Wealth, 59(3), 393-415.

Easterlin, R.A (1974). "Does economic growth improve the human lot? Some Empirical Evidence". In Paul A. David \& Melvin W. Reder (eds.), Nations and Households in Economic Growth. New York: Academic Press.

Esteban, J., \& Ray, D. (1999). Conflict and distribution. Journal of Economic Theory, 87(2), 379-415.

Ferrer-I-Carbonell, A. \& Frijters, P. (2004). How Important Is Methodology for the Estimates of the Determinants of Happiness?. The Economic Journal, 114(497), 641-59.

Foster, J. E., \& Wolfson, M. C. (2010). Polarization and the decline of the middle class: Canada and the US. The Journal of Economic Inequality, 8(2), 247-273.

Frey, B. S, \& Stutzer, A. (2002a). Happiness and Economics : How the Economy and Institutions Affect Wellbeing. Princeton, N.J.: Princeton University Press.

Kobus, M. (2014). Polarization measurement for ordinal data. The Journal of Economic Inequality, 13(2), 275-297.

Lazar, A., \& Silber, J. (2013). On the cardinal measurement of health inequality when only ordinal information is available on individual health status. Health Economics, 22(1), 106-113.

Madden, D. (2011). The Impact of an Economic Boom on the Level and Distribution of Subjective Wellbeing: Ireland, 1994-2001. Journal of Happiness Studies, 12(4), 667-679.

Madden, D. (2010). Ordinal and cardinal measures of health inequality: an empirical comparison. Health Economics, 19(2), 243-250. 
Niimi, Y. (2016). What Affects Happiness Inequality? Evidence from Japan. Journal of Happiness Studies, 1-23.

Ott, J. (2005). "Level and inequality of happiness in nations: Does greater happiness of a greater number imply greater inequality in happiness?" Journal of Happiness Studies, 6(4), 397-420.

Ovaska, T., \& Takashima, R. (2010). Does a rising tide lift all the boats? Explaining the national inequality of happiness. Journal of Economic Issues, 44(1), 205-224.

Schneider, S. M. (2015). Income inequality and subjective wellbeing: Trends, challenges, and research directions. Journal of Happiness Studies, 17(4), 1719-1739.

Silber, J., \& Yalonetzky, G. (2011). Measuring inequality in life chances with ordinal variables. Research on Economic Inequality, 19, 77-98.

Stevenson, B. \& Wolfers, J. (2008). "Happiness inequality in the United States" (No. w14220). National Bureau of Economic Research.

Stiglitz, J.E., Sen, A. \& Fitoussi, J-P (2009). Report by the Commission on the Measurement of Economic Performance and Social Progress. www.stiglitz-sen-fitoussi.fr: CMEPSP.

Van Doorslaer, E., \& Jones, A. M. (2003). Inequalities in self-reported health: validation of a new approach to measurement. Journal of health economics, 22(1), 61-87.

Veenhoven, R. (2005). Return of inequality in modern society? Test by dispersion of life-satisfaction across time and nations. Journal of Happiness Studies, 6(4), 457-487.

Veenhoven, R. (2011). Trend inequality of happiness in nations 1946-2010: How much happiness differs across citizens. Erasmus University Rotterdam. Available at: http://worlddatabaseofhappiness.eur.nl/hap_nat/findingreports/Archive/TrendReport_InequalityHap piness_1946-2010.pdf

Wang, Y.Q., \& Tsui, K.Y. (2000). Polarization orderings and new classes of polarization indices". Journal of Public Economic Theory, 2(3), 349-363.

Zheng, B. (2008). Measuring inequality with ordinal data: a note. Research on Economic Inequality, 16, 177-188.

Zhang, X., \& Kanbur, R. (2001). What difference do polarisation measures make? An application to China. Journal of Development Studies, 37(3), 85-98. 
Property 1. Increased spread. A regressive transfer in the Pigou-Dalton sense from someone below the median to an individual above the median would increase bipolarisation.

For categorical variables, the transfer is characterized as a Median Preserving Spread and this property is represented by the AF-ordering given in Definition 1.

Let $F(x)=\left(F_{1}, \ldots, F_{J-1}, 1\right)$ be the cumulative distribution of the categorical variable $X$ and $f(x)=\left(f_{1}, \ldots, f_{J-}\right.$ $\left.1, f_{J}\right)$ the probability distribution function, where $f_{j}$ is the proportion of population for the category $j$. Let $m$ denote the median category such that $F_{m-1}<1 / 2$ and $F_{m} \geq 1 / 2$.

Definition 1. AF-ordering (Allison and Foster, 2004). Given two cumulative distribution functions $F^{1}$ and $F^{2}, F^{2}$ has greater spread from the median than $F^{1}$, that is $F^{1} \prec_{A F} F^{2}$ if the following conditions are satisfied:

(1) $F^{1}$ and $F^{2}$ have the same median category, $m\left(F^{1}\right)=m\left(F^{2}\right)=m$.

(2) $F_{j}^{1} \leq F_{j}^{2} \forall j<m$.

(3) $F_{j}^{1} \geq F_{j}^{2} \forall j \geq m$.

$F^{1}$ has a greater proportion of population concentrated around the median, hence it is less spread away from the median. We conclude therefore that $F^{1}$ is associated with lower levels of inequality for all possible scales.

Property 2. Increased bipolarity. A progressive Pigou-Dalton transfer on either side of the median would increase polarisation. The transfer decreases the dispersion within the group of population below/above the median, thus fostering the levels of polarisation while decreasing inequality.

Definition 2. Increased bipolarity transfer. There is an increased bipolarity transfer of a proportion $\xi>0$, from the category $j$ to $j+1$ and from $k$ to $k-1$ if $f_{j}, f_{k}>\xi, j+1 \leq k-1$ and $j \geq m$ or $k<m$.

Definition 3. Increased bipolarity ordering (Apouey, 2007). Let $>_{I B}$ define the partial ordering, for any two distributions $F^{1}, F^{2}$, such that $m\left(F^{1}\right)=m\left(F^{2}\right)=m$, we say that $F^{1} \prec_{I B} F^{2}$ if $F^{2}$ is obtained from $F^{1}$ by a finite sequence of IB transfers.

Let $\Phi(F)$ be an order-preserving function of the $>_{A F}$ relation. Let $\widehat{F}$ denote the most egalitarian distribution, which has all the probability mass concentrated at the median category. $\widetilde{F}$ is the most unequal distribution, where half of individuals are in the lowest category and the other half of the population is placed at the best possible state ${ }^{12}$. The family proposed by Abul Naga and Yalcin (2008) is constructed by normalizing $\Phi(F)$ by $\Phi(\widehat{F})$ and $\Phi(\widetilde{F})$, as follows:

$$
\mathrm{I}(F)=\frac{\Phi(F)-\Phi(\widehat{F})}{\Phi(\widetilde{F})-\Phi(\widehat{F})} .
$$

A function of the form $\Phi(F)=\sum_{j<m} g_{1}\left(F_{j}\right)-\sum_{j \geq m} g_{2}\left(F_{j}\right)$ was chosen, which satisfies the AF-ordering when $g_{1}$ and $g_{2}$ are strictly increasing on $F$ (see Lemma 4, Abul Naga and Yalcin (2008)). Replacing $g_{1}\left(F_{j}\right)=\left(F_{j}\right)^{\alpha}$ and $g_{2}\left(F_{j}\right)=\left(F_{j}\right)^{\beta}$ in Eq. (A.1) we get the ANY index in Eq. (1).

The following proposition characterises the ANY measure as an index of polarisation.

Proposition 1. $I_{\alpha, \beta}$ satisfies the partial IB-ordering for $\alpha>1, \beta \in(0,1)$.

Proof.

(1) IB movement above the median.

12 The most equal distribution has all the probability mass in one category: $\widehat{F}=[0,0, \ldots, 1, \ldots, 1]$, $\hat{f}=[0,0, \ldots, 1,0, \ldots, 0]$. Note that $\widehat{F}$ is not unique. The probability mass can cluster around any category, so there are $J$ different most equal distributions. The most unequal distribution concentrates the probability mass around the two ends: $\widetilde{F}=[0.5,0.5, \ldots, 1], \widehat{f}=[0.5,0, \ldots, 0.5]$. 
For any proportions $\left(f_{a}, f_{b}, \xi\right) ; f_{a}, f_{b}, \zeta \geq 0, f_{a} \geq \xi, f_{a}+f_{b}+\zeta \leq 1 / 2$ and $f_{b}+\xi>0$ and for any two distributions $F^{1}, F^{2}$, such that,

$$
\begin{gathered}
m\left(F^{1}\right)=m\left(F^{2}\right) ; m \in[1, J-2], \\
F_{j}^{1}=F_{j}^{2} \text { for } j \in[1, J-3] \text { if } J \geq 4, \\
F_{J-2}^{1}=\frac{1}{2}+f_{a} \text { and } F_{J-1}^{1}=\frac{1}{2}+f_{a}+f_{b} .
\end{gathered}
$$

If $F^{2}$ is obtained from $F^{1}$ by an IB transfer, setting $j=J-2$ and $k=J$, we have:

$$
F_{J-2}^{1}=\frac{1}{2}+f_{a}-\xi \text { and } F_{J-1}^{1}=\frac{1}{2}+f_{a}+f_{b}+\xi .
$$

Then, $I_{\alpha, \beta}\left(F^{1}\right)<I_{\alpha, \beta}\left(F^{2}\right)$ if and only if $\Phi\left(F^{1}\right)<\Phi\left(F^{2}\right)$. Then,

$$
\sum_{j<m} g_{1}\left(F^{1}\right)-\sum_{j \geq m} g_{2}\left(F^{1}\right)<\sum_{j<m} g_{1}\left(F^{2}\right)-\sum_{j \geq m} g_{2}\left(F^{2}\right) .
$$

Since $\sum_{j<m} g_{1}\left(F^{1}\right)=\sum_{j<m} g_{1}\left(F^{2}\right)$,

$$
-\sum_{j \geq m} g_{2}\left(F^{1}\right)<-\sum_{j \geq m} g_{2}\left(F^{2}\right) \rightarrow \sum_{j \geq m} g_{2}\left(F^{1}\right)>\sum_{j \geq m} g_{2}\left(F^{2}\right)
$$

This is equivalent to,

$$
\begin{aligned}
& g_{2}\left(f_{a}\right)+g_{2}\left(f_{a}+f_{b}\right)>g_{2}\left(f_{a}-\xi\right)+g_{2}\left(f_{a}+f_{b}+\xi\right) \\
& g_{2}\left(f_{a}\right)-g_{2}\left(f_{a}-\xi\right)>g_{2}\left(f_{a}+f_{b}+\xi\right)-g_{2}\left(f_{a}+f_{b}\right)
\end{aligned}
$$

The previous statement is true if $g_{2}(F)$ is strictly concave on $\Re_{+}$. Taking $g_{2}(F)=(F)^{\beta}$, the previous result implies that the IB-ordering is satisfied if $\beta \in(0,1)$.

(2) IB movement below the median.

For any proportions $\left(f_{a}, f_{b}, \xi\right) ; f_{a}, f_{b}, \xi \geq 0, \mathrm{a} \geq \xi, f_{a}+f_{b}+\xi \leq 1 / 2$ and $f_{b}+\xi>0$ and for any two distributions $F^{1}, F^{2}$, such that,

$$
\begin{gathered}
m\left(F^{1}\right)=m\left(F^{2}\right) ; m \in[3, J], \\
F_{j}^{1}=F_{j}^{2} \text { for } j \in[4, J] ; J \geq 4, \\
F_{1}^{1}=f_{a} \text { and } F_{2}^{1}=f_{a}+f_{b} .
\end{gathered}
$$

If $F^{2}$ is obtained from $F^{1}$ by an IB transfer, setting $j=1$ and $k=3$, we have:

$$
F_{1}^{1}=f_{a}-\xi \text { and } F_{2}^{1}=f_{a}+f_{b}+\xi .
$$

Again, $I_{\alpha, \beta}\left(F^{1}\right)<I_{\alpha, \beta}\left(F^{2}\right)$ if and only if $\Phi\left(F^{1}\right)<\Phi\left(F^{2}\right)$. Then,

$$
\sum_{j<m} g_{1}\left(F^{1}\right)-\sum_{j \geq m} g_{2}\left(F^{1}\right)<\sum_{j<m} g_{1}\left(F^{2}\right)-\sum_{j \geq m} g_{2}\left(F^{2}\right) .
$$

Given that $\sum_{j \geq m} g_{2}\left(F^{1}\right)=\sum_{j \geq m} g_{2}\left(F^{2}\right)$, this is equivalent to

$$
\sum_{j<m} g_{1}\left(F^{1}\right)<\sum_{j<m} g_{1}\left(F^{2}\right)
$$

which is satisfied if,

$$
\begin{aligned}
& g_{1}\left(f_{a}\right)+g_{1}\left(f_{a}+f_{b}\right)<g_{1}\left(f_{a}-\xi\right)+g_{1}\left(f_{a}+f_{b}+\xi\right) \\
& g_{1}\left(f_{a}\right)-g_{1}\left(f_{a}-\xi\right)<g_{1}\left(f_{a}+f_{b}+\xi\right)-g_{1}\left(f_{a}+f_{b}\right)
\end{aligned}
$$


The previous statement is true if $g_{1}(F)$ is strictly convex on $\Re_{+}$. Taking $g_{1}\left(F_{j}\right)=\left(F_{j}\right)^{\alpha}$, the previous result implies that the IB-ordering is satisfied if $\alpha>1$.

The ANY polarisation measure can be expressed as a weighted sum of the two previous cases as follows,

$$
I_{\alpha, \beta}=\omega_{1} I_{\alpha, 0}+\omega_{2} I_{\infty, \beta}
$$

where

$$
\omega_{1}=\frac{\kappa_{\alpha, 0}+J+1-m}{\kappa_{\alpha, \beta}+J+1-m}, \omega_{2}=\frac{\kappa_{\infty, \beta}+J+1-m}{\kappa_{\alpha, \beta}+J+1-m}
$$

and

$$
I_{\infty, \beta}(F)=\frac{\sum_{j \geq m}\left(F_{j}\right)^{\beta}+J+1-m}{(J-m)\left(1-(1 / 2)^{\beta}\right)}, \beta \in(0,1) ; I_{\alpha, 0}(F)=\frac{\sum_{j<m}\left(F_{j}\right)^{\alpha}}{(m-1)(1 / 2)^{\alpha}}, \alpha>1 .
$$

Analogously, the ANY inequality measure can be also expressed as a weighted sum of the of the two limiting cases:

$$
I_{\alpha, \beta}=\omega_{1} I_{\alpha, \infty}+\omega_{2} I_{0, \beta}-\left(\frac{J-t}{\kappa_{\alpha, \beta}+J+1-m}\right)
$$

where

$$
\omega_{1}=\frac{\kappa_{\alpha, \infty}+J+1-m}{\kappa_{\alpha, \beta}+J+1-m}, \omega_{2}=\frac{\kappa_{0, \beta}+J+1-m}{\kappa_{\alpha, \beta}+J+1-m}
$$

and

$$
I_{0, \beta}(F)=\frac{J-\sum_{j \geq m}\left(F_{j}\right)^{\beta}}{J-1-(J-m)(1 / 2)^{\beta}}, \beta>1 ; I_{\alpha, \infty}(F)=\frac{\sum_{j<m}\left(F_{j}\right)^{\alpha}-t+J+1-m}{(m-1)(1 / 2)^{\alpha}+J-m}, \alpha \in(0,1) .
$$

being $t$ is the number of categories such that $F_{j}=1$.

\section{Statistical inference}

Let $X$ be a categorical variable of $J$ categories and denote by $f_{j}, \forall j=1, \ldots, J$; the proportion of the $j$-th category, such that $\sum_{j=1}^{J} f_{j}=1$. Then, $\left(f_{1}, \ldots, f_{J}\right)$ follows a multinomial distribution with $n$ observations and a vector of probabilities $\left(\Pi_{1}, \ldots, \Pi_{J}\right)$. The covariance matrix of the variable $X$ is then given by

$$
\boldsymbol{\Sigma}=\left(\begin{array}{cccc}
f_{1}\left(1-f_{1}\right) & -f_{1} f_{2} & \ldots & -f_{1} f_{J} \\
-f_{1} f_{2} & f_{2}\left(1-f_{2}\right) & \ldots & -f_{2} f_{J} \\
\vdots & \vdots & \ddots & \vdots \\
-f_{J} f_{1} & -f_{J} f_{2} & \cdots & f_{J}\left(1-f_{J}\right)
\end{array}\right)
$$

The ANY measure is then a nonlinear function of the parameter estimates of $f_{1}, \ldots, f_{J}$. Using the Central Limit Theorem, the index asymptotically follows a normal distribution with a covariance matrix derived by the delta method as,

$$
n \cdot \operatorname{Var}\left(I_{\alpha, \beta}\right)=\mathbf{A} \mathbf{\Sigma} \mathbf{A}^{\prime},
$$

where, $\quad \mathbf{A}=\left(\frac{\partial I_{\alpha, \beta}}{\partial f_{I}}, \ldots, \frac{\partial I_{\alpha, \beta}}{\partial f_{J}}\right)$ and being the $k$-th element of $\mathbf{A}$ given by

$$
\frac{\partial I_{\alpha, \beta}}{\partial f_{k}}=\left(\frac{1}{\kappa_{\alpha, \beta}+J+1-m}\right)\left(\sum_{j=k}^{m-1} \alpha \mathrm{F}_{j}^{\alpha-1}-\sum_{j=m}^{J} \beta F_{j}^{\beta-1}\right),
$$

where $F_{j}$ stands for the cumulative relative frequency. 
Appendix B: Sensitivity analysis.

1

2

3

4

5

6

7

8

9

10

11

12

13

14

15

16

17

18

19

20

21

22

23

24

25

26

27

28

29

30

31

32

33

34

35

36

37

38

39

40

41

42

43

44

45

46

47

48

49

50

51

52

53

54

55

56

57

58

59

60

61

62

63

64

65

[Figure A1 HERE] 
Fig. 1 Increased spread (left) and increased bipolarity (right) in life satisfaction
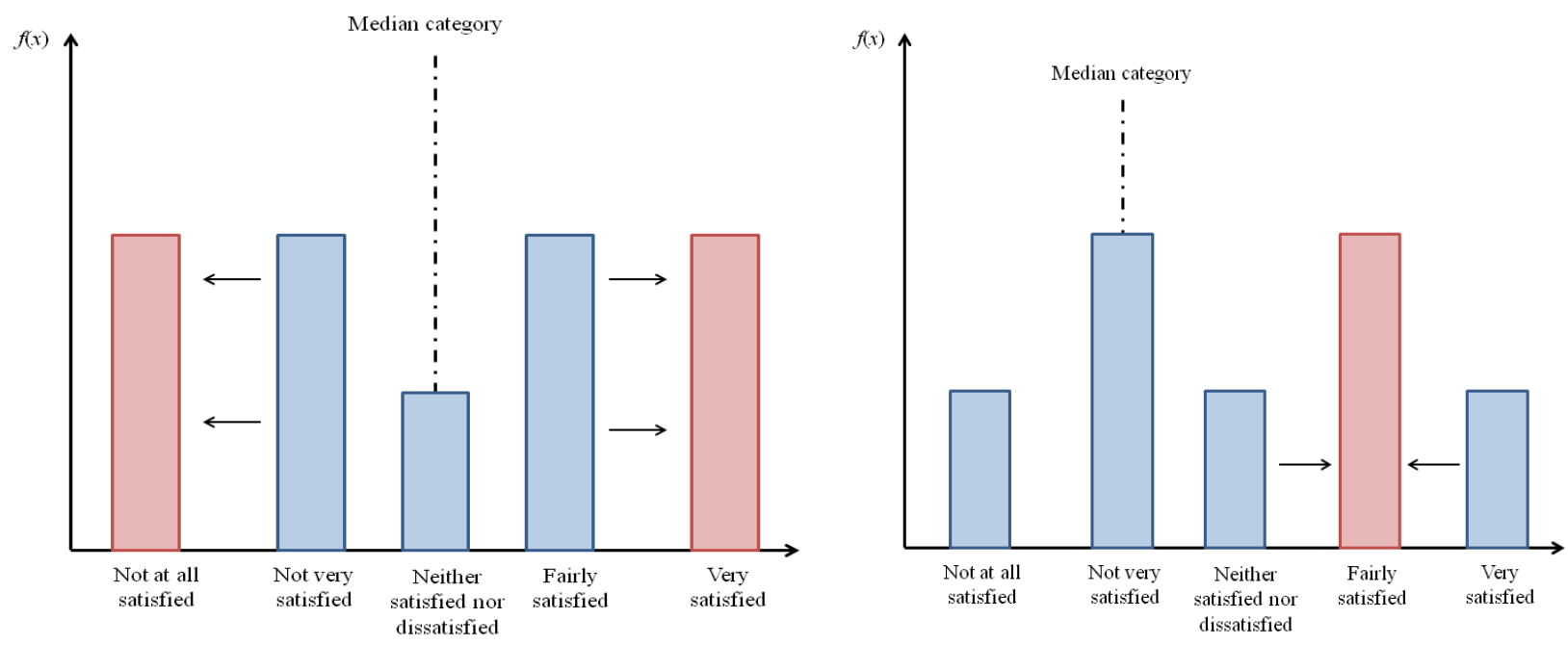
Fig. 2 Evolution of inequality in life satisfaction in Europe. 1995-2014

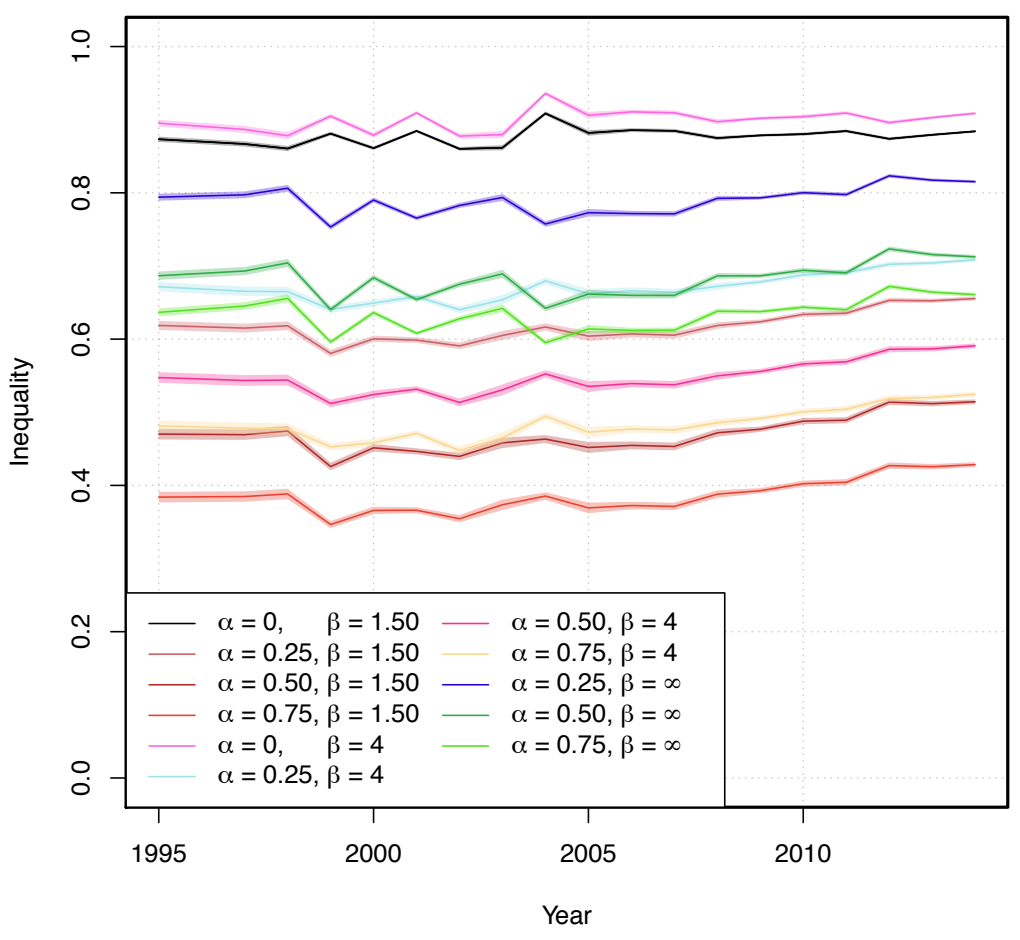


Fig. 3 Evolution of polarisation in life satisfaction in Europe. 1995-2014

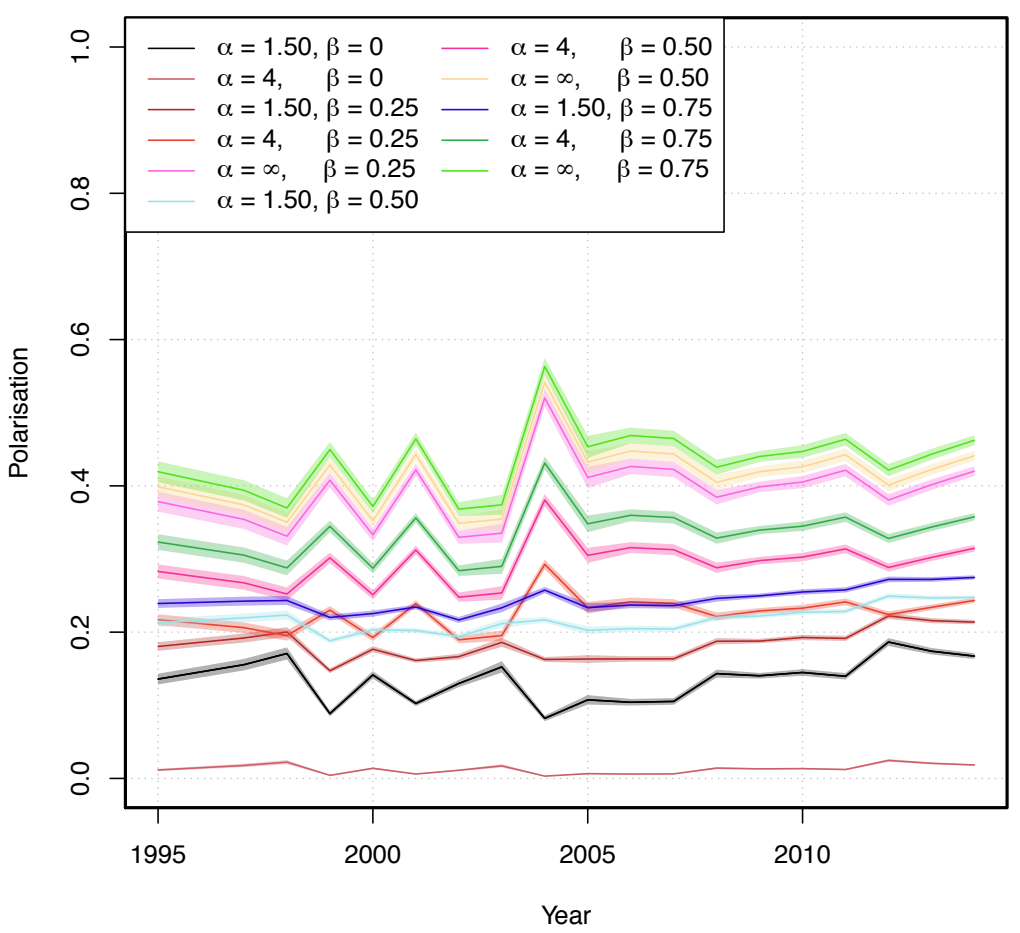


Fig. 4 Evolution of inequality and polarisation in life satisfaction
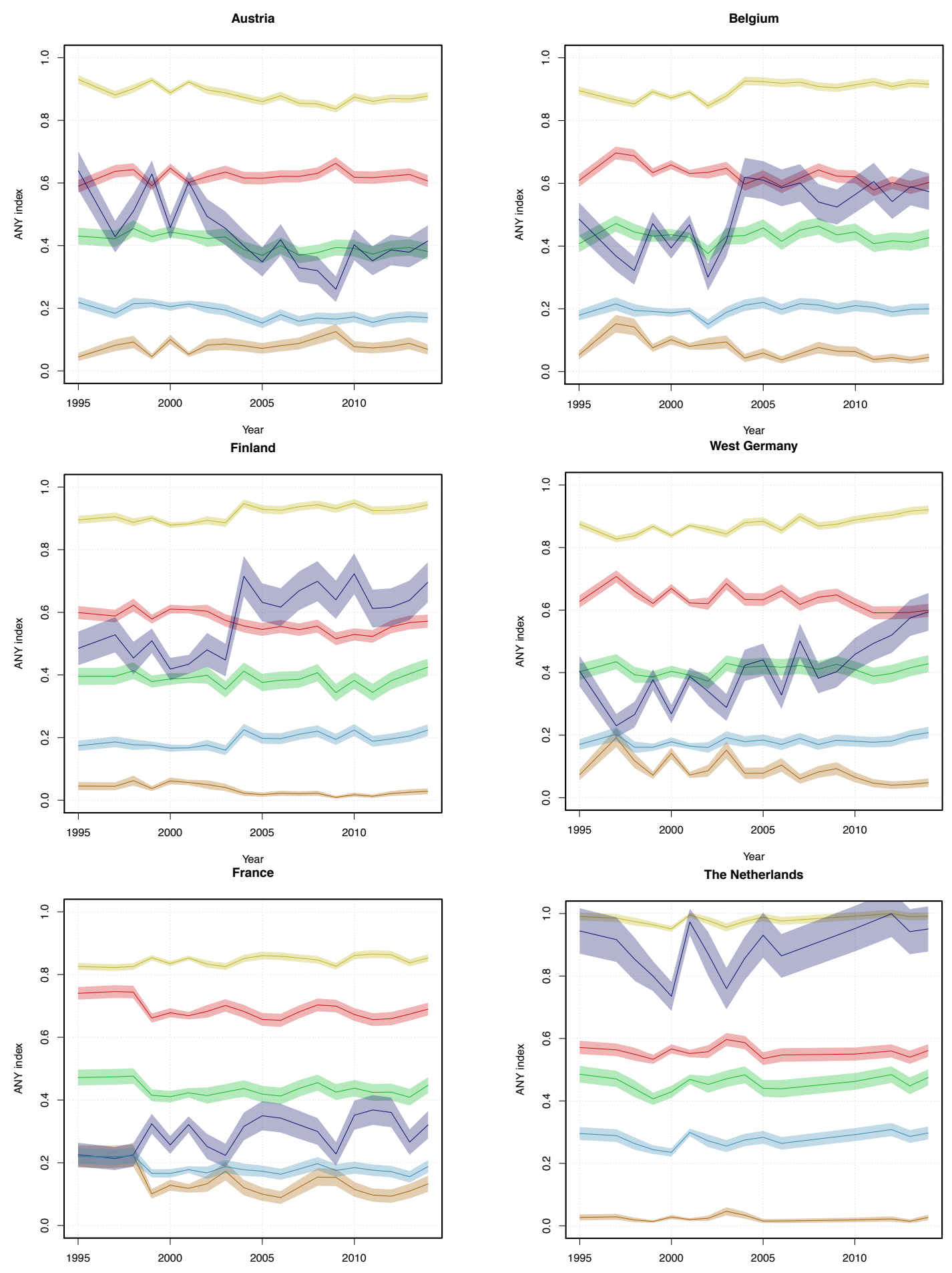

\begin{tabular}{lll} 
Year & & \multicolumn{1}{c}{ Year } \\
\hline $\begin{array}{l}\alpha=0.50, \beta=\infty \\
\alpha=0, \quad \beta=1.50\end{array}-\begin{array}{l}\alpha=0.50, \beta=1.50 \\
\alpha=1.50, \beta=0\end{array}$ & $-\alpha=\infty, \quad \beta=0.50$ \\
$\alpha=1.50, \beta=0.50$
\end{tabular}


Fig. 4 continued

Fig. A1 Evolution of inequality and polarisation in life satisfaction
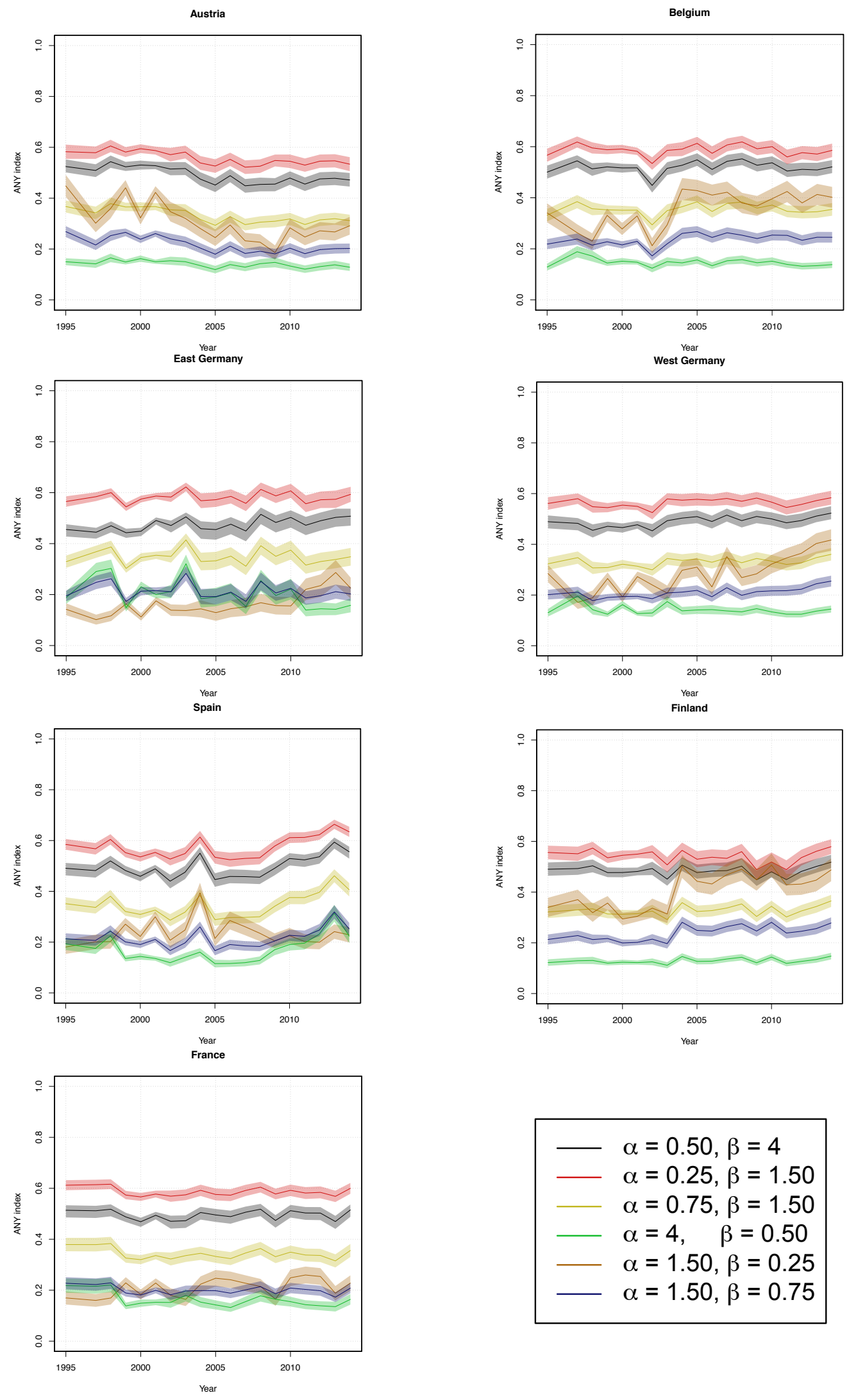
Fig. A1 Continued
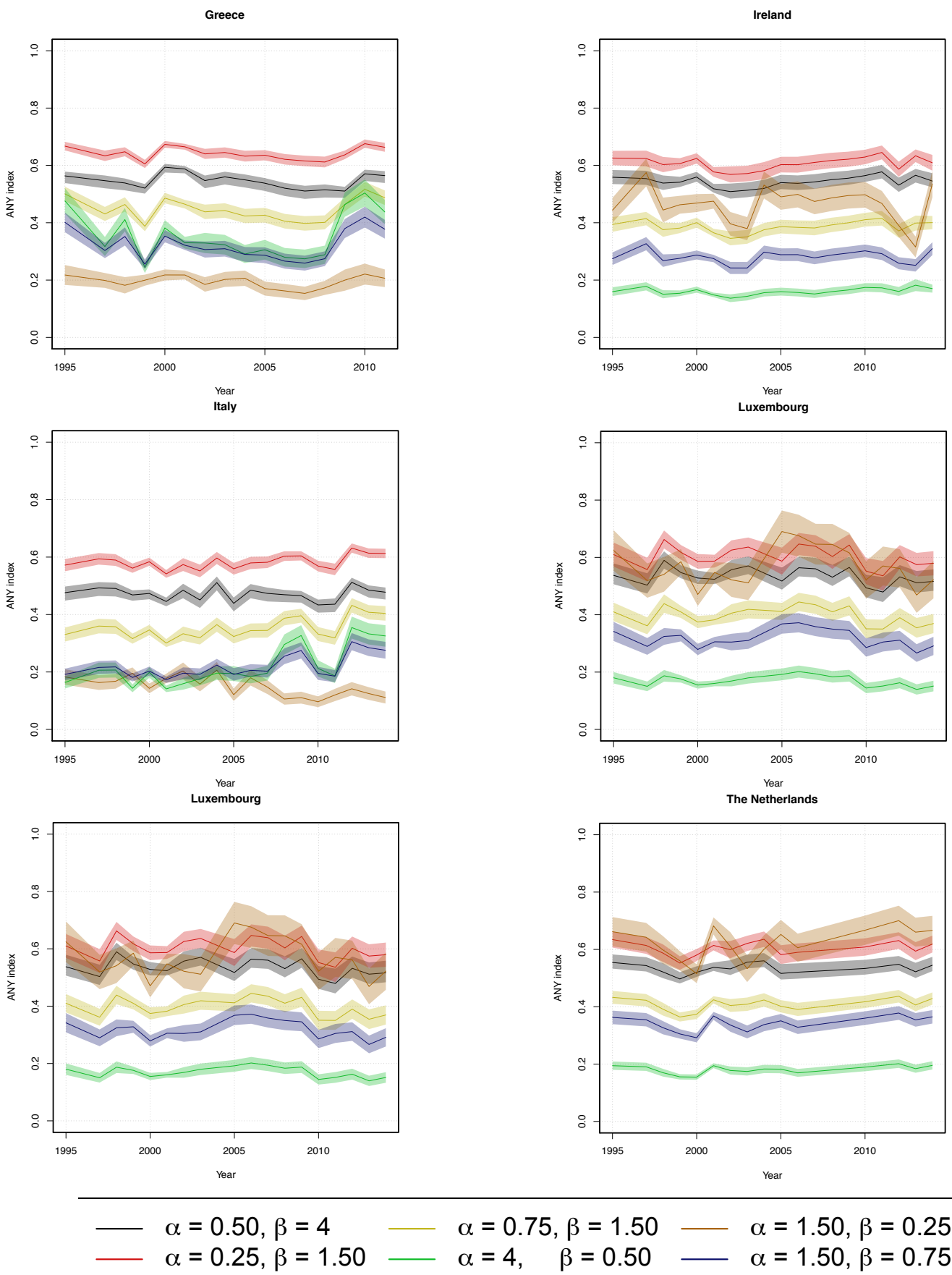

$\alpha=0.75, \beta=1.50$

$\alpha=1.50, \beta=0.25$

$\alpha=0.25, \beta=1.50$

$\alpha=4, \quad \beta=0.50$

$\alpha=1.50, \beta=0.75$ 
Table 1. Effect of different scales of life satisfaction on mean and inequality levels

\begin{tabular}{|c|c|c|c|c|c|c|c|}
\hline & $\begin{array}{c}\text { not at all } \\
\text { satisfied }\end{array}$ & $\begin{array}{l}\text { not very } \\
\text { satisfied }\end{array}$ & $\begin{array}{c}\text { neither satis. } \\
\text { nor dissat. }\end{array}$ & $\begin{array}{c}\text { fairly } \\
\text { satisfied }\end{array}$ & $\begin{array}{c}\text { very } \\
\text { satisfied }\end{array}$ & mean & $\mathrm{CV}$ \\
\hline Scale $(c)$ & 1 & 2 & 3 & 4 & 5 & & \\
\hline$f_{1}$ & 0.2 & 0.2 & 0.2 & 0.2 & 0.2 & 3 & 0.47 \\
\hline$f_{2}$ & 0.4 & 0.1 & 0.1 & 0.1 & 0.3 & 2.8 & 0.61 \\
\hline Scale $\left(c^{\prime}\right)$ & 1 & 2 & 3 & 4 & 50 & & \\
\hline$f_{1}$ & 0.2 & 0.2 & 0.2 & 0.2 & 0.2 & 12 & 1.58 \\
\hline$f_{2}$ & 0.4 & 0.1 & 0.1 & 0.1 & 0.3 & 16.3 & 1.35 \\
\hline
\end{tabular}


Table 2. Sensitivity of the ANY measure to IB movements

\begin{tabular}{rrrr|r|rrrrrrr}
\hline & $c_{1}$ & \multicolumn{1}{c}{$c_{2}$} & \multicolumn{1}{c}{$c_{3}$} & \multicolumn{1}{c|}{$c_{4}$} & \multicolumn{1}{c}{$c_{5}$} & \multicolumn{1}{c}{$c_{6}$} & $c_{7}$ & $A(0.5)$ & $I_{2,2}$ & $I_{2,0.5}$ & $I_{0.5,2}$ \\
\hline$f_{1}$ & 0.15 & 0.1 & 0.15 & 0.2 & 0.15 & 0.1 & 0.15 & & & & \\
$F_{1}$ & 0.15 & 0.25 & 0.4 & 0.6 & 0.75 & 0.85 & 1 & 0.3363 & 0.5333 & 0.4190 & 0.6576 \\
& & & & & & & & & & & \\
$f_{2}$ & 0.05 & 0.3 & 0.05 & 0.2 & 0.15 & 0.1 & 0.15 & & & & \\
$F_{2}$ & 0.05 & 0.35 & 0.4 & 0.6 & 0.75 & 0.85 & 1 & 0.3442 & 0.5467 & 0.4436 & 0.6411 \\
& & & & & & & & & & & \\
$f_{3}$ & 0.15 & 0.1 & 0.15 & 0.2 & 0.05 & 0.3 & 0.05 & & & & \\
$F_{3}$ & 0.15 & 0.25 & 0.4 & 0.6 & 0.65 & 0.95 & 1 & 0.3442 & 0.5200 & 0.4233 & 0.6485 \\
\hline
\end{tabular}


Table 3. Change in life satisfaction inequality over the period $1973-2014^{(1)}$

\begin{tabular}{|c|c|c|c|c|c|c|c|}
\hline & $\begin{array}{l}\Delta \text { ANY index } \\
\alpha=0.5, \beta=\infty\end{array}$ & $\begin{array}{l}\Delta \text { ANY index } \\
\alpha=0, \beta=1.5\end{array}$ & $\begin{array}{l}\Delta \text { ANY index } \\
\alpha=0.5, \beta=1.5\end{array}$ & $\begin{array}{l}\Delta \text { ANY index } \\
\alpha=0.25, \beta=1.5\end{array}$ & $\begin{array}{l}\Delta \text { ANY index } \\
\alpha=0.5, \beta=4\end{array}$ & $\Delta$ Gini index & $\begin{array}{l}\Delta \text { Standard } \\
\text { deviation }\end{array}$ \\
\hline Austria & $0.0166(2.81 \%)$ & $-0.0533(-5.73 \%)$ & $-0.0490(-11.39 \%)$ & $-0.0489(-8.39 \%)$ & $-0.0528(-10.07 \%)$ & $-0.0031(-3.05 \%)$ & $-0.0304(-4.68 \%)$ \\
\hline $1995-2014$ & {$[-0.0114,0.0444]$} & {$[-0.0718,-0.0344]$} & {$[-0.0859,-0.0125]$} & {$[-0.0884,-0.0100]$} & {$[-0.0876,-0.0190]$} & {$[-0.0122,0.0059]$} & {$[-0.0733,0.0125]$} \\
\hline Belgium & $0.0327(5.74 \%)$ & $-0.0665(-6.76 \%)$ & $-0.0470(-9.93 \%)$ & $(-6.29 \%)$ & $-0.0272(-4.95 \%)$ & 0.004 & $0.0057(0.87 \%)$ \\
\hline $1973-2014$ & {$[0.0038,0.0611]$} & {$[-0.0844,-0.0484]$} & {$[-0.0844,-0.0092]$} & {$[-0.0765,-0.0005]$} & $+, 0.0051]$ & {$[-0.0040,0.0135]$} & $25,0.0543]$ \\
\hline East Germany & $-0.0111(-1.59 \%)$ & $0.0502(6.27 \%)$ & $0.0515(13.28 \%)$ & 0.0547 & $(23.16 \%)$ & & $0.0878(14.05 \%)$ \\
\hline $1990-2014$ & {$[-0.0431,0.0195]$} & {$[0.0330,0.0679]$} & {$[0.0119,0.0908]$} & {$[0.0206, \mathrm{C}$} & $.1373]$ & {$[-0$} & {$[0.0299,0.1448]$} \\
\hline West Germany & $52 \%)$ & $3.67 \%)$ & 0.04 & $\%)$ & 0.0 & 0.0 & \\
\hline $1973-2014$ & {$[-0.0675,-0.017]$} & {$[0.0570,0.0898]$} & {$[0.0120,0.0783]$} & {$[0.0130,0.0801]$} & $0.0991]$ & $0.0089]$ & {$[-0.0133,0.0695]$} \\
\hline Spain & $-0.0169(-2.25 \%)$ & $-3.52 \%)$ & $10.75 \%)$ & $.05 \%)$ & $17 \%)$ & -0.0 & -0. \\
\hline $1985-2014$ & {$[-0.0453,0.0124]$} & {$[-0.0490,-0.0133]$} & $.0251]$ & $.0206]$ & $3,-0.0342]$ & {$[-0.0$} & {$[-0.1181,-0.0192]$} \\
\hline Finland & $-0.0276(-4.61 \%)$ & $0.0479(5.35 \%)$ & $7.36 \%)$ & 0.022 & 0.02 & -0.0 & 0.0017 \\
\hline $1995-2014$ & {$[-0.0579,0.0018]$} & {$[0.0293,0.0664]$} & {$[-0.0109,0.0684]$} & {$[-0.0186,0.0628]$} & {$[-0.0074,0.0630]$} & $0.0088]$ & {$[-0.0472,0.0503]$} \\
\hline France & $-0.0079(-1.14 \%)$ & $(1.64 \%)$ & 0.00 & $3 \%)$ & $0 .($ & -0.0 & $\%)$ \\
\hline $1973-2014$ & {$[-0.0316,0.0168]$} & {$[-0.0009,0.0280]$} & {$[-0.0233,0.0393]$} & $0.0385]$ & $, 0.0510]$ & , 0.0108] & {$[-0.026,0.0644]$} \\
\hline Greece & $-0.0361(-4.09 \%)$ & $-0.0574(-6.68 \%)$ & $-0.1159(-17.09 \%)$ & $-0.0959(-12.63 \%)$ & $-0.1570(-21.76 \%)$ & $-0.0499(-22.17 \%)$ & $-0.2155(-20.79 \%)$ \\
\hline $1981-2011$ & {$[-0.0621,-0.0102]$} & {$[-0.0724,-0.0417]$} & {$[-0.1465,-0.0849]$} & {$[-0.1175,-0.0738]$} & {$[-0.1914,-0.1214]$} & {$[-0.0641,-0.0354]$} & {$[-0.2583,-0.172]$} \\
\hline Ireland & $-0.0424(-6.75 \%)$ & $0.0075(0.79 \%)$ & $-0.0401(-8.02 \%)$ & $-0.0428(-6.57 \%)$ & $-0.0370(-6.35 \%)$ & $-0.0120(-10.56 \%)$ & $-0.0694(-9.47 \%)$ \\
\hline $1975-2014$ & {$[-0.0690,-0.0173]$} & {$[-0.0088,0.0239]$} & {$[-0.0732,-0.0081]$} & {$[-0.0773,-0.0118]$} & {$[-0.0644,-0.0108]$} & {$[-0.0200,-0.0040]$} & {$[-0.1119,-0.0271]$} \\
\hline Italy & $0.0377(4.93 \%)$ & $-0.0213(-2.64 \%)$ & $0.0169(3.56 \%)$ & $0.0082(1.35 \%)$ & $-0.0147(-2.98 \%)$ & $0.0082(5.95 \%)$ & $0.0123(1.69 \%)$ \\
\hline $1973-2014$ & {$[0.0141,0.0609]$} & {$[-0.0312,-0.0109]$} & {$[-0.0111,0.0452]$} & {$[-0.0126,0.0292]$} & {$[-0.0468,0.0178]$} & {$[-0.0037,0.0202]$} & {$[-0.0269,0.0519]$} \\
\hline Luxembourg & $-0.0306(-5.17 \%)$ & $-0.0108(-1.12 \%)$ & $-0.0497(-10.46 \%)$ & $-0.0464(-7.42 \%)$ & $-0.0404(-7.25 \%)$ & $-0.0108(-10.51 \%)$ & $-0.0569(-8.42 \%)$ \\
\hline $1973-2014$ & {$[-0.0791,0.0192]$} & {$[-0.0403,0.0201]$} & {$[-0.1107,0.0129]$} & {$[-0.1121,0.0226]$} & {$[-0.0910,0.0118]$} & {$[-0.0246,0.0025]$} & {$[-0.1338,0.0196]$} \\
\hline The Netherlands & $0.0029(0.53 \%)$ & $0.0361(3.77 \%)$ & $0.0498(11.69 \%)$ & $0.0463(8.09 \%)$ & $0.0289(5.60 \%)$ & $0.0012(1.23 \%$ & $0.0224(3.61 \%)$ \\
\hline $1973-2014$ & {$[-0.0239,0.0290]$} & {$[0.0193,0.0529]$} & {$[0.0148,0.0835]$} & {$[0.0052,0.0858]$} & {$[0.0007,0.0563]$} & {$[-0.0056,0.0081]$} & {$[-0.0164,0.061]$} \\
\hline Portugal & $-0.0102(-1.18 \%)$ & $0.0005(0.07 \%)$ & $-0.0112(-2.08 \%)$ & $-0.009(-1.40 \%)$ & $-0.0089(-1.79 \%)$ & $-0.0081(-4.80 \%)$ & $-0.0344(-4.38 \%)$ \\
\hline $1985-2014$ & {$[-0.0359,0.0154]$} & {$[-0.0075,0.0083]$} & {$[-0.0416,0.0192]$} & {$[-0.0296,0.0114]$} & {$[-0.0413,0.0239]$} & {$[-0.0221,0.0059]$} & {$[-0.0743,0.0052]$} \\
\hline
\end{tabular}

(he first and the last 